\title{
Asymptotische Darstellungen der hypergeometrischen Funktionen für große Werte eines Parameters
}

\author{
E. WAGNER
}

Die in bekannten Tabellenwerken (z. B. BatEMAN/ERDÉLYI: Higher transcendental functions I. New York 1953) angegebenen asymptotischen Entwicklungen der hypergeometrischen Funktion $F^{\prime}(a, b, c ; z)$ für $|b| \rightarrow \infty$ bei festen Werten von $a, c, z$ sind fehlerhaft. In der vorlięgenden Aribeit werden asymptotische Darstellungen von $F$ für $a$ (komplex) $\rightarrow \infty$ bei beliebigen, festen Werten von $b, c(c \neq 0,-1,-2, \ldots)$ und $z(z \neq 0,|\operatorname{Arg}(1-z)|<\pi)$ hergeleitet. Aus ihnen ergeben sich durch Vertauschung von $a$ und $b$ entsprechende asymptotische Darstellungen von $F$ für $|b| \rightarrow \infty$.

Находящиеся в общеизвестных таблицах (напр. Бейтмен/Әрдейи: Высшие трансцендентные функции І. Москва 1973) асимптотические разложения гипергеометрической функции $F(a, b, c ; z)$ при $|b| \rightarrow \infty$ и постоянных зпачениях $a, c, z$ ошибочны. В настоящей работе выводятся асимптотические прецставления функции $F(a, b, c ; z)$ при $a$ (комплексно) $\rightarrow \infty$ и любых постоянных значеншях $b, c(c \neq 0,-1,-2, \ldots)$ и $z(z=0$, $|\operatorname{Arg}(1-z)|<\pi)$. Нз них перестановкоі $a$ п $b$ получаютсл соответствующие асимптотические предстваления функции $F$ при $|b| \rightarrow \infty$.

Asymptotic expansions of the hypergeometric function $F(a, b, c ; z)$ for $|b| \rightarrow \infty$ where $a, c, z$ are fixed complex numbers given in well-known tables (e.g. Bateman/Erdélyt: Higher transcendental functions I. New York 1953) are incorrect. In the present paper asymptotic representations of the hypergeometric function $F$ for $a$ (complex) $\rightarrow \infty$ are derived where $b, c(c \neq 0,-1,-2, \ldots)$ and $z(z \neq 0,|\operatorname{Arg}(1-z)|<\pi)$ are fixed complex numbers. By change of $a$ and $b$ appropriate asymptotic representations of $F$ for $|b| \rightarrow \infty$ are obtained.

Asymptotische Darstellungen der hypergeometrischen Funktionen $F(a, b, c ; z)$ für $|b| \rightarrow \infty$ bei festen Werten der übrigen Parameter $a, c$ und der Variablen $z$ sind in [1] und [2] angegeben, aber man prüft anhand geeigneter funktionaler Bezichungen für $F$ leicht nach, daß diese Darstellungen unter den dort angegebenen Voraussetzungen nicht richtig sein können. $\mathrm{DaB}$ die in [2] skizzierte Herleitung dieser asymptotischen Darstellungen nicht stichhaltig ist, liegt offenbar an einer ungerechtfertigten Vertauschung zweier Grenzprozesse.

In der vorliegenden Arbeit werden zunächst asymptotische Darstellungen von $F^{\prime}(a, b, c ; z)$ für $|a| \rightarrow \infty$ bei festen Werten von $b, c, z$ hergeleitet, aus denen sich wegen der bekannten Beziehung $F(a, b, c ; z)=F(b, a, c ; z)$ sofort asymptotische Darstellungen fiir $|b| \rightarrow \infty$ bei festen Werten von $a, c, z$ ergeben.

Im folgenden verwenden wir die Bezeichnungen

$$
a=a_{1}+i a_{2}=|a| e^{i \alpha} \quad \operatorname{mit} \quad \alpha=\operatorname{Arg} a \in(-\pi, \pi]
$$

(entsprechend für $b, c$ ) und

$$
z=x+i y=|z| e^{i \omega} \quad \text { mit } \quad \omega=\operatorname{Arg} z \in(-\pi, \pi]
$$

und setzen voraus, $d a ß$

$$
z \neq 0 \text { und }|\operatorname{Arg}(1-z)|<\pi
$$


ist. Unter Potenzen komplexer Zahlen sind, falls nichts anderes vereinbart ist, ihre Hauptwerte zu verstehen, so daß insbesondere gilt

$$
z^{a}=\exp \left\{a_{1} \ln |z|-a_{2} \omega+i\left[a_{1} \omega+a_{2} \ln |z|\right]\right\} .
$$

Satz 1: Unter den Voraussetzungen (3) gelten bei beliebigen, festen Werten 'b, $c$ $(c \neq 0,-1,-2, \ldots)$ und $z$ für $|a| \rightarrow \infty$ die asymptotischen Darstellungen

$$
\begin{aligned}
\frac{1}{\Gamma(c)} F(a, b, c ; z)= & \frac{(-a z)^{-b}}{\Gamma(c-b)}[1+o(1)] \\
& +\frac{e^{ \pm \pi i(b-c)}}{\Gamma(b)}(1-z)^{c-a-b}(-a z)^{b-c}[1+o(1)] .
\end{aligned}
$$

In den Fällen

1. $y>0$ und $a_{1} \geqq 0$,

2. $z<0$ und $a_{2}<0$,

3. $y<0$ und $a_{1}<0$,

4. $0<z<1$ und $a_{2} \geqq 0$ ist im Exponenten das Pluszeichen und arg $(-a z)$ aus dem Intervall $\left(-\frac{3 \pi}{2}, \frac{\pi}{2}\right) \approx u$
nehmen. In den restlichen Fällen
5. $y>0$ und $a_{1}<0$,
6. $z<0$ und $a_{2} \geqq 0$,
7. $y<0$ und $a_{1} \geqq 0$,
8. $0<z<1$ und $a_{2}<0$ ist im Exponenten das Minuszeichen und $\arg (-a z)$ aus dem Intervall $\left(-\frac{\pi}{2}, \frac{3 \pi}{2}\right) z \cdot u$
nehmen.

Beweis: Ausgehend von der bekannten Integraldarstellung

$$
\frac{F(a, b, c ; z)}{\Gamma(c)}=\frac{1}{\Gamma(b) \Gamma(c-b)} \int_{0}^{1} \frac{t^{b-1}(1-t)^{c-b-1}}{(1-t z)^{a}} d t \quad\left(c_{1}>b_{1}>0\right)
$$

führen wir den Beweis unter den einschränkenden Bedingungen

$$
c_{1}>b_{1}>0
$$

zuerst für $y>0$ (d. h. $0<\omega<\pi)$ und dann für $z<0(\omega=\pi)$. Anschließend wird unter Verwendung bekannter Funktionalgleichungen für die hypergeometrischen Funktionen gezeigt, daß die Zusatzvoraussetzung (7) weggelassen und der Gültigkeitsbereich von (5) auf alle komplexen $z$, die (3) genügen, ausgeweitet werden kann. Zur Abkürzung bezeichnen wir den Integranden in (6) mit $f=f(a, b, c ; z, t)$.

1. Schritt: Beweis von (5) für $y>0, a_{1} \geqq 0$ unter den Bedingungen (7):

Es sei $\varphi$ ein Winkel, der den Bedingungen

und

$$
|\varphi| \leqq \pi / 4
$$

bzw.

$$
\omega-\operatorname{Arg}(z-1)<\varphi<0 \text { für } a_{2} \geqq 0
$$

$$
0<\varphi<\omega \text { für } a_{2}<0
$$

genügt, $R$ eine Konstante mit

$$
R>\max (1 /|z|,|z-1| /|z|),
$$


sowie

$$
\eta=-\lambda e^{i \varphi / z}, \quad \delta=\lambda(z-1) e^{i \varphi / z}, \quad \lambda=|a|^{-3 / 4} .
$$

Dann gilt für den in Abb. 1 dargestellten Integrationsweg nach dem Cauchyschen Integralsatz

$$
\int_{0}^{1} f d t=\sum_{v=1}^{5} \int_{\mathbb{C}_{v}} f d t
$$

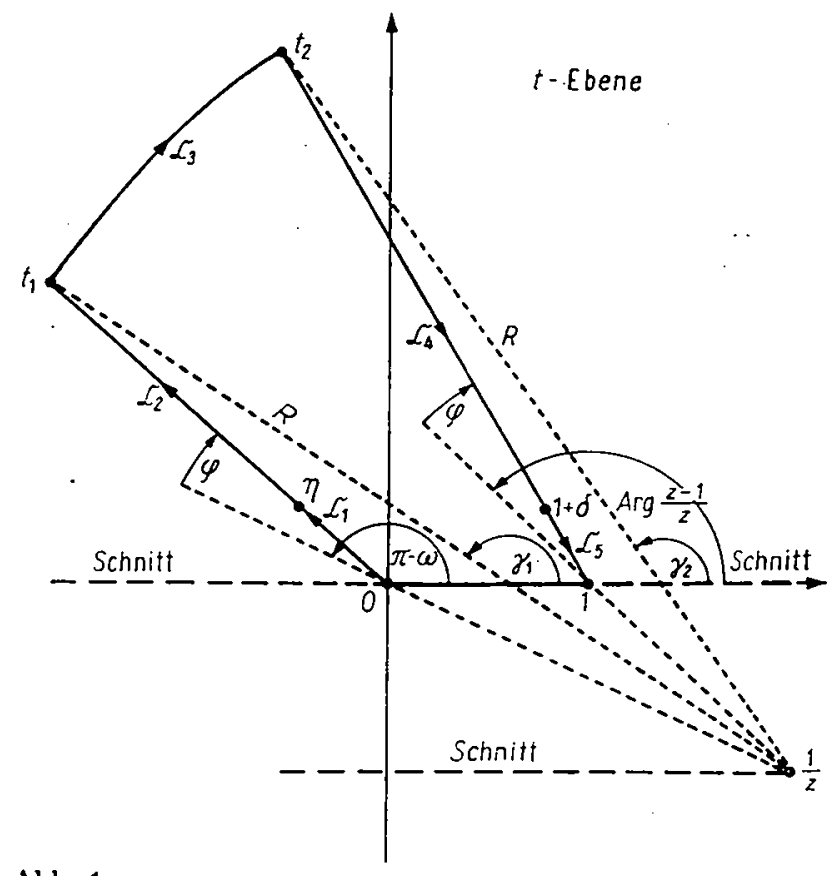

Abb. 1

1.1. Asymptotische Darstellung des Integrals über $\mathfrak{C}_{1}$

\section{Wegen}

und

$$
0 \leqq|t| \leqq|\eta|=\lambda /|z|=o(1)
$$

$$
0 \leqq\left|a t^{2}\right| \leqq|a||\eta|^{2}=|a|^{-1 / 2} /|z|^{2}=o(1)
$$

gilt gleichmäßig für alle $t \in \mathfrak{S}_{1}$ und $|a| \rightarrow \infty$

und

$$
(1-t)^{c-b-1} \sim 1
$$

$$
(1-t z)^{-a}=\exp \{-a \log (1-t z)\}=\exp \left\{a t z+O\left(a t^{2}\right)\right\} \sim \exp (a t z) .
$$

Setzt man noch atz $=-\tau$, so erhält man

$$
\int_{\mathcal{S}_{1}} f d t \sim \int_{0}^{-a z \eta}\left(\frac{\tau}{-a z}\right)^{b-1} e^{-\tau}(-a z)^{-1} d \tau .
$$


Aus (9) und (10) folgt

$$
\operatorname{Arg}\left(\frac{\tau}{-a z}\right)=\operatorname{Arg} \tau-\alpha-\operatorname{Arg}(-z)=\varphi+\pi-\omega \in(0, \pi),
$$

so daß man schreiben kann

$$
\int_{\mathfrak{S}_{1}} f d t \sim a^{-b}(-z)^{-b} \int_{0}^{-a z \eta} e^{-\tau} \tau^{b-1} d \tau
$$

Bekanntlich strebt das Integral auf der rechten Seite für $|a| \rightarrow \infty$ wegen

$$
-a z \eta=|a|^{1 / 4} e^{i(\alpha+\varphi)}
$$

und der aus den Bedingungen (8), (9), (10) sich ergebenden Ungleichungen

$$
\frac{-\pi}{4} \leqq \varphi \leqq \alpha+\varphi \leqq \frac{\pi}{2}+\varphi<\frac{\pi}{2} \quad \text { für } \quad a_{2} \geqq 0
$$

bzw.

$$
\frac{-\pi}{2}<\frac{-\pi}{2}+\varphi \leqq \alpha+\varphi<\varphi \leqq \frac{\pi}{4} \quad \text { für } \quad a_{2}<0
$$

gegen $\Gamma(b)$, so daß man erhält

$$
\int_{\mho_{1}} f d t \sim \Gamma(b) a^{-b}(-z)^{-b}=\Gamma(b)(-a z)^{-b} \quad\left(\frac{-3 \pi}{2}<\arg (-a z)<\frac{\pi}{2}\right) .
$$

\subsection{Asymptotische Darstellung des Integrals über $\mathfrak{C}_{5}$}

Es ist

$$
\int_{\mathbb{C}_{3}} f d t=\int_{0}^{-\delta}(1-\tau)^{b-1} \tau^{c-b-1}(1-z+\tau z)^{-a} d \tau
$$

mit geradlinig verlaufendem Integrationsweg von 0 bis $-\delta$. Wegen

$$
0 \leqq|\tau| \leqq|\delta|=|a|^{-3 / 4}|z-1| /|z|=o(1)
$$

erhält man wie unter 1.1. gleichmäßig fïr alle $\tau$

$$
(1-\tau)^{b-1} \tau^{c-b-1}(1-z+z \tau)^{-a} \sim \tau^{c-b-1}(1-z)^{-a} \exp \left(\frac{-a z \tau}{1-z}\right) \quad(|a| \rightarrow \infty)
$$

und daraus

$$
\int_{\mathfrak{S}_{1}} f d t \sim(1-z)^{-a} \int_{0}^{-\frac{a z \delta}{1-z}}\left(\frac{1-z}{a z} u\right)^{c-b-1} e^{-u}\left(\frac{1-z}{a z}\right) d u .
$$

Aus (9) und (10) folgt

$\operatorname{Arg}\left(\frac{1-z}{a z} u\right)=\operatorname{Arg}(1-z)-\alpha-\omega+\operatorname{Arg} u=\operatorname{Arg}(1-z)-\omega+\varphi \in(-\pi, 0)$, und unter Berïcksichtigung von

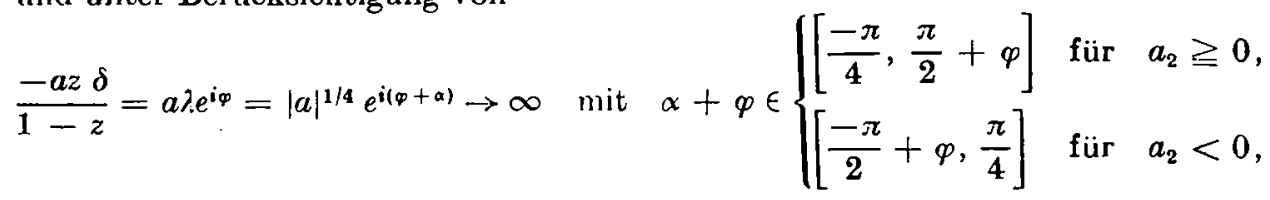


wobei $\frac{\pi}{2}+\varphi<\frac{\pi}{2}$ für $a_{2} \geqq 0$ und $\frac{-\pi}{2}+\varphi>\frac{-\pi}{2}$ für $a_{2}<0$ ist, crgibt sich wie unter 1.1.

$$
\int_{\mathfrak{C}_{3}} f d t \sim \Gamma(c-b)(1-z)^{c-b-a} a^{b-c} z^{b-c}
$$

bzw.

$$
\int_{\mathscr{E}_{0}} f d t \sim \Gamma(c-b) e^{i \pi(b-c)}(1-z)^{c-0-o}(-a z)^{b-c} \quad\left(\arg (-a z) \in\left(\frac{-3 \pi}{2}, \frac{\pi}{2}\right)\right) .
$$

1.3. Abschätzung des Integrals über $\mathbb{E}_{2}$

Für $t \in \mathbb{C}_{2}$ ist

$$
t^{b-1}=O\left(|t|^{b_{1}-1}\right)=O\left(\max \left(1, \lambda^{b_{1}-1}\right)\right)
$$

und, da $\mathfrak{\mho}_{2}$ wegen (9) und (10) nicht durch $t=1$ geht,

$$
(1-t)^{c-b-1}=O(1)
$$

Mit der Parameterdarstellung $t=-\lambda e^{i_{p}} \tau / z, 1 \leqq \tau \leqq\left|t_{1}\right||z| / \lambda$ erhält man

$$
\left\{(1-t z)^{-a} \mid=\exp \left\{-|a|\left[\cos \alpha \ln \left|1+\lambda e^{i \varphi} \tau\right|-\sin \alpha \operatorname{Arg}\left(1+\lambda e^{i \varphi} \tau\right)\right]\right\}\right. \text {. }
$$

Man sieht leicht, daß unter der Einschränkung (8) $\ln \left|1+\lambda \cdot e^{i \varphi} \tau\right|$ eine monoton wachsende Funktion von $\tau$ und $\operatorname{Arg}\left(1+\lambda e^{i_{\varphi}} \tau\right)$ monoton wachsend für $\varphi>0$ und monoton fallend für $\varphi<0$ ist, so daß

und

$$
\ln \left|1+\lambda e^{i \varphi} \tau\right| \geqq \ln \left|1+\lambda \cdot e^{i \varphi}\right|
$$

$$
\operatorname{Arg}\left(1+\lambda e^{\left.i \varphi_{\tau}\right)} \begin{cases}\leqq \operatorname{Arg}\left(1+\lambda e^{i_{\varphi}}\right) & \text { für } \varphi<0 \\ \geqq \operatorname{Arg}\left(1+\lambda e^{i_{\digamma}}\right) & \text { für } \varphi>0\end{cases}\right.
$$

ist. Für $|a| \rightarrow \infty$ strebt $\lambda \rightarrow 0$, und es gilt

$$
\begin{aligned}
& \ln \left|1+\lambda \cdot e^{i \varphi}\right|=\lambda \cos \varphi+O\left(\lambda^{2}\right) \\
& \operatorname{Arg}\left(1+\lambda e^{i \varphi}\right)=\arctan \frac{\lambda \cdot \sin \varphi}{1+\lambda \cdot \cos \varphi}=i \sin \varphi+O\left(\lambda^{2}\right) .
\end{aligned}
$$

Beachtet man noch, daß wegen (9) und (10) $\alpha$ und $p$ entgegengesetzte Vorzeichen besitzen, so folgt aus (22) die Abschätzung

$\left|(1-t z)^{-a}\right| \leqq \exp \left\{-|a|^{1 / 4} \cos (\alpha+\varphi)+o(1)\right\} \leqq \exp \left\{-|a|^{1 / 4} \sin |\varphi|+o(1)\right\}$.

Daraus folgt

$$
\int_{\varepsilon_{3}} f d t=O\left(\max \left(1, \lambda^{b_{1}-1}\right)\right) \exp \left\{-|a|^{1 / 4} \sin |\varphi|\right\}=O\left(\int_{\mathbb{E}_{1}} f d t\right) .
$$




\subsection{Abschätzung des Integrals über $\mathfrak{C}_{4}$ :}

Wie unter 1.3. sieht man, daß für $t \in \mathfrak{C}_{4}$

und

$$
t^{b-1}=O(1), \quad(1-t)^{c-b-1}=O\left(\max \left(1, \lambda^{c_{1}-b_{1}-1}\right)\right)
$$

$\left|(1-\ell z)^{-a}\right|=\left|(1-z)^{-a}\right|\left|\left(1+\lambda e^{i \varphi} \tau\right)^{-a}\right| \leqq\left|(1-z)^{-a}\right| \exp \left\{-|a|^{1 / 4} \sin |\varphi|+o(1)\right\}$

gilt, so daß man wegen (20)

$$
\int_{\sigma_{0}} f d t=o\left(\int_{\sigma_{0}} f d t\right)
$$

erhält.

\subsection{Abschätzung des Integrals über $\mathfrak{c}_{3}$}

Der Integrationsweg $\mathfrak{F}_{3}$ ist ein Kreisbogen mit einem hinreichend großen, von $a, b$ und $c$ unabhängigen Radius $R$ (vgl. (11)) und dem Mittelpunkt $1 / z$, so da $B$ auf $\mathfrak{S}_{3}$

$$
t^{b-1}=O(1) \quad \text { und } \quad(1-t)^{c-b-1}=O(1)
$$

ist. Mit der Parameterdarstellung (vgl. Abb. 1)

$$
t=1 / z+R e^{i \gamma}, \quad 0<\gamma_{2} \leqq \gamma_{.} \leqq \gamma_{1}<\pi \quad\left(\gamma_{v}=\operatorname{Arg}\left(t_{\nu}-1 / z\right)\right)
$$

ist

$$
\left|(1-t z)^{-a}\right|=\exp \{-|a|[\cos \alpha \ln |R z|-(-\pi+\gamma+\omega) \sin \alpha]\} .
$$

Aus Abb. 1 ist unter Berücksichtigung von (9) und (10) zu ersehen, daB

und

$$
\gamma \leqq \gamma_{1}<\pi-\omega \text { für } a_{2} \geqq 0
$$

$$
\gamma \geqq \gamma_{2}>\operatorname{Arg}(z-1)-\omega \text { für } a_{2}<0
$$

ist, so daß die Abschätzungen

$$
(-\pi+\gamma+\omega) \sin \alpha \leqq\left\{\begin{array}{l}
-\mu_{1} \sin \alpha \text { für } 0 \leqq \alpha \leqq \frac{\pi}{2} \\
\left(\mu_{2}+\operatorname{Arg}(1-z)\right) \sin \alpha \text { für } \frac{-\pi}{2} \leq \alpha<0
\end{array}\right.
$$

mit

$$
\mu_{1}=\pi-\gamma_{1}-\omega>0 \text { und } \mu_{2}=\gamma_{2}+\omega-\operatorname{Arg}(z-1)>0
$$

gelten. Aus (25), (26) und (27) folgt

$$
\begin{aligned}
& \int_{\mathfrak{C}_{2}} f d t \\
& =\left\{\begin{array}{l}
O\left(\exp \left\{-|a|\left[\cos \alpha \ln |R z|+\mu_{1} \sin \alpha\right]\right\}\right) \quad \text { für } 0 \leqq \alpha \leqq \frac{\pi}{2} \\
O\left(\exp \left\{-|a|\left[\cos \alpha \ln |R z|-\left(\mu_{2}+\operatorname{Arg}(1-z)\right) \sin \alpha\right]\right\}\right) \quad \text { für } \quad \frac{-\pi}{2} \leqq \alpha<0,
\end{array}\right.
\end{aligned}
$$


so daß wegen (11), (16), (20) und (28)

gilt.

$$
\int_{\mathfrak{V}_{3}} f d t= \begin{cases}o\left(\int_{\mathbb{E}_{1}} f d t\right) & \text { für } a_{2} \geqq 0 \\ o\left(\int_{\mathfrak{E}_{1}} f d t\right) & \text { für } a_{2} \leqslant 0\end{cases}
$$

Zusammenfassend erhält man aus (6), (13), (16), (21), (23), (24) und (29) die Behauptung von Satz 1 im 1. Fall unter den einschränkenden Bedingungen (7).

2. Schritt: Beweis von (5) für $a_{1} \geqq 0, z<0$ unter den Bedingungen (7):

Man geht wieder von der Integraldarstellung (6) aus und ersetzt den Integrationsweg durch ein komplexes Kurvenintegral in der $t$-Ebene gemä $B$ Abb. 2 . $R, \delta$ und $\eta$ wählen wir wie in (11) und (12), $\varphi$ sei konstant mit

$$
\begin{aligned}
& -\frac{\pi}{4} \leqq \varphi<0 \quad \text { für } a_{2} \geqq 0, \\
& 0<\varphi \leqq \frac{\pi}{4} \quad \text { für } \quad a_{2}<0 .
\end{aligned}
$$

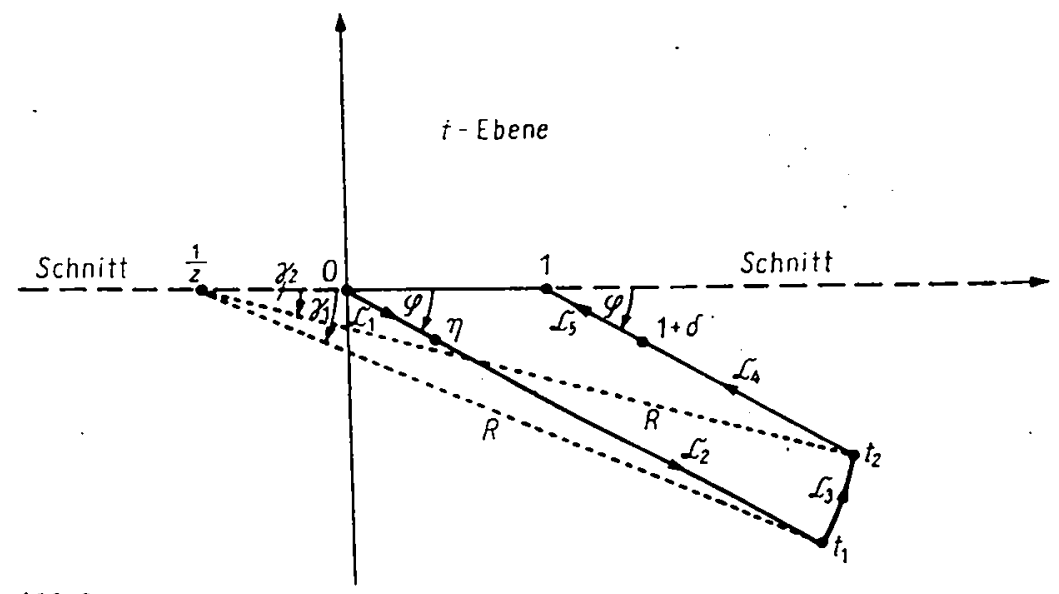

Abb.2

2.1. Man überzeugt sich leicht, daß man wie unter 1.1, mit nur geringfügigen Modifikationen, die asymptotische Darstellung

$$
\int_{E_{2}} f d t \sim \Gamma(b) a^{-b}(-z)^{-b}=\Gamma(b)(-a z)^{-b} \quad\left(\frac{-\pi}{2} \leqq \arg (-a z)=\alpha \leqq \frac{\pi}{2}\right)
$$

erhält.

2.2. Wie unter 1.2. erhält man auch jetzt die asymptotische Gleichung (19), aber hier ist

$$
\operatorname{Arg}\left(\frac{1-z}{a z} u\right)=\operatorname{Arg}(1-z)-\operatorname{Arg}(-a z)+\operatorname{Arg} u \pm \pi=\varphi \pm \pi,
$$

wobei das + für $a_{2} \geqq 0$ und das Minuszeichen für $a_{2}<0$ steht, so daß aus (19)

$\int_{c_{s} .} f d t \sim e^{ \pm \pi i(b-c)}(1-z)^{c-b-a}(-a z)^{b-c} \quad\left(\frac{-\pi}{2} \leqq \arg (-a z)=\propto \leqq \frac{\pi}{2}\right)$

mit + für $\iota_{2}<0$ und dem Minuszeichen für $a_{2} \geqq 0$ folgt. 
2.3. Man überprüft leicht, daß die unter 1.3. und 1.4. angegebenen Rechnungen auch für $z<0$ gültig sind, so daß die Beziehungen (23) und (24) gelten.

2.4. Auf $\mathfrak{E}_{3}$ gilt offenbar (25). Mit der Parameterdarstellung $t=1 / z+R e^{i \gamma}$ ist

$$
\left|(1-t z)^{-a}\right|=\exp \{-|a|[\cos \alpha \ln |R z|-\gamma \sin \alpha]\} \text {. }
$$

Der Parameter $\gamma$ genügt den Ungleichungen (vgl. Abb. 2)

so daß man

$$
\begin{array}{lll}
\varphi<\gamma_{1} \leqq \gamma \leqq \gamma_{2}<0 & \text { für } \quad a_{2} \geqq 0 \quad \text { bzw. } \quad \alpha \in\left[0, \frac{\pi}{2}\right], \\
0<\gamma_{2} \leqq \gamma \leqq \gamma_{1}<\varphi & \text { für } \quad a_{2}<0 \text { bzw. } \quad \alpha \in\left[\frac{-\pi}{2}, 0\right),
\end{array}
$$

$$
\left|(1-t z)^{-a}\right| \leqq \exp \left\{-|a|\left[\cos \alpha \ln |R z|-\gamma_{2} \sin \alpha\right]\right\}
$$

erhält. Aus (25), (33) und (34) ergibt sich für ein hinreichend großes $R$

$$
\int_{\mathfrak{G}_{2}} f d t=O\left(\exp \left\{-|a|\left[\cos \alpha \ln |R z|-\gamma_{2} \sin \alpha\right]\right\}\right)=o\left(\int_{\mathbb{C}_{3}} f d t\right) \text {. }
$$

Zusammenfassend erhält man aus (6), (13), (23), (24), (32), (33) und (34) die Formel (5) in den Fällen 2 und 6, aber zunächst nur unter den einschränkenden Bedingungen $a_{1} \geqq 0$ und $(7)$.

3. Schritt: Beweis von (5) im Fall 5 unter den Bedingungen (7):

Dazu gehen wir von der bekannten Funktionalgleichung

$$
F(a, b, c ; z)=(1-z)^{c-a-b} F(c-a, c-b, c ; z)
$$

aus. Aus $a_{1}<0$ und (7) folgen $c_{1}>c_{1}-b_{1}>0$ und $c_{1}-a_{1}>0$, so da $\beta$ in die rechte Seite der Gleichung (35) die für diesen Fall bereits bewiesene Formel (5) eingesetzt werden kann. Man erhält

$$
\begin{aligned}
\frac{F(a, b, c ; z)}{\Gamma(c)}= & (1-z)^{c-a-b}\left\{\frac{[-(c-a) z]^{b-c}}{\Gamma(b)}[1+o(1)]\right. \\
& \left.+\frac{e^{-x b i}}{\Gamma(c-b)}(1-z)^{a-c+b}[-(c-a) z]^{-b}\{1+o(1)]\right\} .
\end{aligned}
$$

Ụnter Beriicksichtigung von

und

$$
[-(c-a) z]^{b-c} \sim(a z)^{b-c}=e^{-x i(b-c)}(-a z)^{b-c} \quad\left(\arg (-a z) \in\left(\frac{-\pi}{2}, \frac{3 \pi}{2}\right)\right)
$$

$$
e^{-x b i}[-(c-a) z]^{-b} \sim e^{-\pi b i}(a z)^{-b}=(-a z)^{-b} \quad\left(\arg (-a z) \in\left(\frac{-\pi}{2}, \frac{3 \pi}{2}\right)\right)
$$

folgt aus (36) sofort die Gültigkeit von (5) im Fall 5 unter den Bedingungen (7).

4. Schritt: Beweis von (5) unter den Bedingungen (7) in den Fallen 2 und 6 , für $a_{1}<0$ :

Wie im 3. Schritt erhält, man durch Finsetzen von (5) in die rechte Seite von (35) wieder die Gleichung (5), wobei fiir $a_{2}>c_{2}$ das Minuszeichen steht und arg (-az) $\epsilon\left(-\frac{\pi}{2}, \frac{3 \pi}{2}\right)$ ist, während fuir $a_{2} \leqq c_{2}$ das Pluszeichen und arg $(-a z)$ aus dem Intervall $\left(\frac{-3 \pi}{2}, \frac{\pi}{2}\right)$ zu nehmen ist. Ist $a_{2}$ beschränkt, so muß $a_{1} \rightarrow-\infty$ streben. 
Dabei strebt

$$
\arg (-a z) \rightarrow\left\{\begin{array}{rll}
\pi & \text { für } & a_{2}>c_{2} \\
-\pi & \text { für } & a_{2} \leqq c_{2}
\end{array}\right.
$$

so daß in beiden Fällen

$$
e^{ \pm \pi i(b-c)}(-a z)^{b-c} \sim|a z|^{b-c}
$$

ist. Demnach ist die Trennung beider Fälle statt durch $c_{2}$ auch durch jede andere Konstante, insbesondere also auch durch 0 möglich. Damit ist der Beweis von (5) für die Fälle 2 und 6 unter den Bedingungen (7) erbracht.

5. Schritt: Beweis von (5) unter den Bedingungen (7) in den restlichen Fällen 3 , 4, 7 und 8:

Bekanntlich ist

$$
F(a, b, c ; z)=(1-z)^{-a} F\left(a, c-b, c ; \frac{z}{z-1}\right) .
$$

Die Funktion $w=z /(z-1)$ bildet die untere $z$-Halbebene in die obere $w$-Halbebene und das Intervall $0<z<1$ auf die negativ-reelle Achse der $w$-Ebene ab. Deshalb folgt die Gültigkeit von (5) in den Fällen 3, 4, 7 und 8 aus ihrer Gültigkeit in den Fällen 5 bzw. 6 bzw. 1 bzw. 2, indem man (5) in die rechte Seite von (37) einsetzt. Auf diese einfache Rechnung kann hier verzichtet werden.

6. Schritt: Eliminierung der Zusatzvoraussetzungen (7):

Nach [3] 9.137, 18. ist

$$
c F(a, b, c ; z)=(c-a) F(a, b, c+1 ; z)+a F(a+1, b, c+1 ; z) .
$$

Wir ersetzen $b$ durch $b+1$ und $c$ durch $c+1$, dividieren durch $\Gamma(c+2)$ und erhalten

$$
\begin{aligned}
\frac{F(a, b+1, c+1 ; z)}{\Gamma(c+1)}= & (c+1-a) \frac{F(a, b+1, c+2 ; z)}{\Gamma(c+2)} \\
& +a \frac{F(a+1, b+1, c+2 ; z)}{\Gamma(c+2)}
\end{aligned}
$$

Setzt man diese Gleichung in die durch $\Gamma(c+2)$ dividierte Gleichung 9.137, 15, al1s [3]:

$$
\frac{F(a, b, c ; z)}{\Gamma(c)}=c \frac{F(a, b+1, c+1 ; z)}{\Gamma(c+1)}-a(c-b) z \frac{F(a+1, b+1, c+2 ; z)}{\Gamma(c+2)},
$$

ein, so erhält man

$$
\begin{aligned}
\frac{F(a, b, c ; z)}{\Gamma(c)}= & c(c+1-a) \frac{F(a, b+1, c+2 ; z)}{\Gamma(c+2)} \\
& +a[c-(c-b) z] \frac{F(a+1, b+1, c+2 ; z)}{\Gamma(c+2)}
\end{aligned}
$$

Für $c_{1}>b_{1}-1$ und $b_{1}>-1$ können in die rechte Seite von (38) die asymptotischen Darstellungen (5) eingesetzt werden. Nach kurzer Rechnung ergibt sich

$$
\begin{aligned}
\frac{F(a, b, c ; z)}{\Gamma(c)}= & \frac{(-a z)^{-b}}{\Gamma(c-b+1)}[c-b+o(1)] \\
& +\frac{e^{ \pm \pi i(b-c)}}{\Gamma(b+1)}(1-z)^{c-a-b}(-a z)^{b-c}[b+o(1)] .
\end{aligned}
$$


Sind $c-b \neq 0$ und $b \neq 0$, so ist diese Gleichung äquivalent (5). Durch $n$-malige Wiederholung des Verfahrens beweist man die Gültigkeit von (5) für $b_{1}>-n$, $c_{1}>b_{1}-n, b \neq 0,-1,-2, \ldots,-n+1$ und $c-b \neq 0,-1,-2, \ldots,-n+1$. Da $n$ beliebig gro $B$ sein kann, gilt (5) für jeden festen Wert von $b$ und $c$, aber unter der vorläufigen Einschränkung, da $B$ und $c-b$ keine nichtpositiven ganzen Zahlen sind. Von dieser Einschränkung kann man sich wie folgt leicht befreien. Wenn $b$ eine nichtpositive ganze $\mathrm{Zahl}$ ist, so ist $F$ ein Polynom $(-b)$-ten Grades in $z$ :

$$
\begin{aligned}
\frac{F(a, b, c ; z)}{\Gamma(c)}= & \sum_{\nu=0}^{-b} \frac{a(a+1) \cdots(a+v-1) b(b+1) \cdots(b+v-1)}{\Gamma(c) c(c+1) \cdots(c+v-1) \nu !} z^{v} \\
& \sim \frac{(-a z)^{-b}}{\Gamma(c-b)} \quad(|a| \rightarrow \infty),
\end{aligned}
$$

also ist (5) auch in diesem Fall richtig. Ist $c-b$ eine nichtpositive ganze Zahl, so folgt aus (35) und der eben hergeleiteten Darstellung (39)

$$
\begin{aligned}
\frac{F(a, b, c ; z)}{\Gamma(c)}= & (1-z)^{c-a-b} \frac{[-(c-a) z]^{b-c}}{I^{\prime}(b)} \\
& \sim(1-z)^{c-a-b} \frac{(a z)^{b-c}}{\Gamma(b)} \quad(|a| \rightarrow \infty)
\end{aligned}
$$

in Úbereinstimmung mit (5). Offenbar können nicht gleichzeitig $b$ und $c-b$ nichtpositive ganze Zahlen sein, denn sonst müßte auch $c$ eine nichtpositive ganze Zahl sein, und das wurde in Satz 1 ausgeschlossen. Damit ist der Beweis von Satz 1 vollständig erbracht.

Ergänzende Bemerkungen: Ist $b \leqq 0$ und ganzzahlig oder $c-b \leqq 0$ und ganzzahlig, kann wegen $1 / \Gamma(b)=0$ bzw. $1 / \Gamma(c-b)=0$ jeweils einer der beiden Summanden in (5) gestrichen werden, und man erhält (39) bzw. (40). Auch wenn weder $b$ noch $c-b$ gleich Null oder einer negativen ganzen $\mathrm{Zahl}$ sind, können leioht Bedingungen angegeben werden, unter denen einer der beiden Summanden in (5) gegenüber dem anderen vernachlässigbar ist. Offenbar kann der erste Summand weggelassen werden, wenn $(1-z)^{-a}$ für $|a| \rightarrow \infty$ exponentiell wächst. Andererseits kann der zweite Summand gestrichen werden, wenn $(1-z)^{-a}$ für $|a| \rightarrow \infty$ exponentiell gegen Null strebt. Wegen

$$
\left|(1-z)^{-a}\right|=\exp \left\{-a_{1} \ln |1-z|+a_{2} \operatorname{Arg}(1-z)\right\}
$$

ljegt exponentielles Wachstum vor, wenn gilt

oder

$$
a_{1}=O(1) \text { und } a_{2} \rightarrow\left\{\begin{array}{lll}
+\infty, & \text { falls } y<0, \\
-\infty, & \text { falls } y>0
\end{array}\right.
$$

$$
a_{2}=O(1) \text { und } a_{1} \rightarrow\left\{\begin{array}{lll}
+\infty, & \text { falls } & 0<z<1 \\
-\infty, & \text { falls } z<0
\end{array}\right.
$$

Andererseits strebt $(1-z)^{-a}$ exponentiell gegen Null, wenn gilt:

oder

$$
a_{1}=O(1) \text { und } a_{2} \rightarrow\left\{\begin{array}{lll}
+\infty, & \text { falls } & y>0 \\
-\infty, & \text { falls } & y<0
\end{array}\right.
$$

$$
a_{2}=O(1) \text { und } a_{1} \rightarrow\left\{\begin{array}{lll}
+\infty, & \text { falls } & z<0 \\
-\infty, & \text { falls } & 0<z<1
\end{array}\right.
$$


Man sieht leicht, daB unter den Bedingungen (I) und (II) aus Satz 1

$$
\frac{F(a, b, c ; z)}{\Gamma(c)} \sim \frac{\left(1-z^{c-a-b}(a z)^{b-c}\right.}{\Gamma(b)} \quad(\arg (a z) \in(-\pi, \pi))
$$

folgt, während unter den Bedingungen (III) und (IV)

$$
\frac{F(a, b, c ; z)}{\Gamma(c)} \sim \frac{(-a z)^{-b}}{\Gamma(c-b)} \quad(\arg (-a z) \in(-\pi, \pi))
$$

gilt. Damit ist gleichzeitig gezeigt, daß die der Unterscheidung der einzelnen Fälle in Satz 1 dienenden Negativitäts- bzw. Nichtnegativitätsforderungen bezüglich $a_{1}$ und $a_{2}$ zu Beschränktheitsbedingungen nach oben bzw. nach unten abgeschwächt werden können. In der Formulierung des folgenden Satzes 2, der sich aus Satz 1 einfach durch Vertauschung der Parameter $a$ und $b$ und aus der bekannten Beziehung $F(a, b, c ; z)=F(b, a, c ; z)$ ergibt, wird diese Tatsache berücksichtigt. Wie üblich bedeutet $b_{*}=O_{R}(1)$, da $B, b$, nach oben beschränkt ist, und entsprechend $\dot{b},=O_{L}(1)$ Beschränktheit von $b$, nach unten.

Satz 2: Unter der Voraussetzung (3) gelten bei festem z und beliebigen, festen Werten von $a$ und $c(c \neq 0,-1,-2, \ldots)$ für $|b| \rightarrow \infty$ die asymptotischen Darstellungen

$$
\begin{aligned}
\frac{F(a, b, c ; z)}{\Gamma(c)}= & \frac{(-b z)^{-a}}{\Gamma(c-a)}(1+o(1)) \\
& +\frac{e^{ \pm \pi i(a-c)}}{\Gamma(a)}(1-z)^{c-a-b}(-b z)^{a-c}(1+o(1)) .
\end{aligned}
$$

Dabei ist in folgenden Füllen das Pluszeichen und arg $(-b z) \in\left(-\frac{3 \pi}{2}, \frac{\pi}{2}\right)$ zu nehmen:
1. $y>0$,
$b_{1}=O_{L}(1)$
2. $z<0$,
$b_{2}=O_{R}(1)$
3. $y<0, \quad b_{1}=O_{R}(1)$;
4. $0<z<1, \quad b_{2}=O_{L}(1)$.

In den folgenden Fällen ist das Minuszeichen und arg $(-b z) \in\left(-\frac{\pi}{2}, \frac{3 \pi}{2}\right)$ zu nehmen:
5. $y>0$,
$b_{1}=O_{R}(1)$
6. $z<0$,
$b_{2}=O_{L}(1)$
7. $y<0$,
$b_{1}=O_{L}(1)$
8. $0<z<1, \quad b_{2}=O_{R}(1)$.

\section{LITERATUR}

[1] Arramowitz, M., and I. A. Steadr: Handbook of Mathematical Functions. New York 1865.

[2] Bateman, H., and A. Erdf́cyr: Higher Transcendental Functions. Vol. 1. New York 1953.

[3] Ryshuk, I. M., und I. S. Gradsters: Summen, Produkt- und Integraltafeln. (Ubers. aus dem Russ.) 2. Aufl. Berlin 1963.

Manuskripteingang: 09. 03. 1981

\section{VERFASSER :}

Dr. sc. E. WAarer

Sektion Mathematik der Martin-Luther-Universität

DDR - 4010 Halle, Universitiitsplatz 6 